\title{
Tanycytic ependymoma of filum terminale: Clinical characteristics and surgical outcomes
}

\author{
ZONGGANG HOU* ${ }^{*}$ XIAOGANG TAO* ${ }^{*}$ JUNTING ZHANG, ZHEN WU and BAIYUN LIU \\ Department of Neurosurgery, Beijing Tiantan Hospital, Capital Medical University, Beijing 100050, P.R. China
}

Received December 25, 2016; Accepted November 29, 2017

DOI: $10.3892 / \mathrm{ol} .2018 .9531$

\begin{abstract}
Tanycytic ependymoma (TE), a rare subtype of ependymoma, was classified as grade II ependymoma by the World Health Organization in 2000 and 2007. Preoperative diagnosis of TE is challenging due to its similarities to schwannoma and astrocytoma; therefore, differentiation is required. The present study investigated the clinical, imaging and pathological characteristics of TE in the filum terminale. A retrospective analysis was conducted on the clinical, imaging, pathological and immunohistochemical characteristics of 8 patients with TE in the filum terminale and the relevant literature was reviewed. Of the 8 patients 7 were female and 1 was male, with an age range of 24-62 years old. The primary clinical symptom observed was lumbago, accompanied by lower limb pain and numbness. Magnetic resonance imaging predominantly identified isointensity on thoracic (T) 1-weighted images and iso- or hyperintense signal intensity on T2-weighted images, with homogeneous or inhomogeneous enhancement. All patients underwent resection of the tumor through a posterior median approach, and total resection was acighieved in 7 patients. During postoperative follow-up, all patients experienced improvement compared with their preoperative status, and were without tumor recurrence. The present study comprised the largest group of cases with TE in the filum terminale reported so far, to the best of our knowledge, which could foster a better understanding of this disease. Complete surgical resection of the tumor has the greatest effectiveness of any treatment for TE in the filum terminale. Postoperative
\end{abstract}

Correspondence to: Professor Baiyun Liu, Department of Neurosurgery, Beijing Tiantan Hospital, Capital Medical University, 6 Tiantan Xili, Dongcheng, Beijing 100050, P.R. China

E-mail: liubaiyun1963@163.com

*Contributed equally

Abbreviations: TE, tanycytic ependymoma; WHO, World Health Organization; MRI, magnetic resonance imaging; JOA, Japanese Orthopedic Association; GTR, gross total resection; GFAP, glial fibrillary acidic protein; EMA, epithelial membrane antigen; $\mathrm{T}$, thoracic

Key words: spinal tumor, tanycytic ependymoma, filum terminale histological examination, immunohistochemistry and electron microscopy for tumor specimens may assist in its diagnosis and differential diagnosis.

\section{Introduction}

Ependymoma is an uncommon type of primary brain tumor, accounting for $37 \%$ of all brain tumors (1). A rare form of ependymoma known as tanycytic ependymoma (TE) has been classified as Grade II by the World Health Organization (WHO) (2). It was first described and termed by Friede and Pollak in 1978 (3). The majority of TE originates from the ependymal cells of the brain ventricular wall and is characterized by elongated spindle cells. Tanycyte cells are implicated in two types of CNS tumors, namely, tanycytic ependymoma and astroblastoma (4). They are elongated spindly bipolar cells that generally present in the circumventricular organs, particularly in the third ventricle and central canal of spinal cord (5). It is common for TE to be misdiagnosed as a nervous system tumor of the spindle cells (6). The definitive diagnosis of TE requires pathological analysis, including histological characteristics. Electron microscopy may contribute to diagnosis and differential diagnosis. Several cases have been diagnosed as astrocytoma or subependymoma or hemangioblastoma by preoperative imaging examination (7). TE is usually located within the cervical and thoracic (T) spinal cord; however, on rare occasions it can be located within the filum terminale. To the best of our knowledge, only 5 cases of TE associated with the filum terminale have been reported in previous studies (Table I) (6,8-11); however, in the present study, 8 cases of TE in the filum terminale were reported. Combined with data from previous studies, the clinical, radiological and pathological features of TE in the filum terminale were summarized for diagnosis and differential diagnosis.

\section{Materials and methods}

The present retrospective study investigated clinical data from 8 patients who underwent microsurgery for a TE of the filum terminale between August 2011 and June 2016 at the Department of Neurosurgery of Beijing Tiantan Hospital (Beijing, China). All patients had performed preoperative and postoperative magnetic resonance imaging (MRI) with gadolinium-contrast enhancement. The relevant clinical data (including patient age, sex, symptoms, neuroimaging, 
preoperative diagnosis, surgical records and follow-up data) were collected via a chart review and telephone interviews. The diagnosis of TE was confirmed by a review of the pathology slides at the Beijing Neurosurgical Institute (Beijing, China). All patients underwent laminectomies to remove the tumor. The following definitions were used: Gross total resection (GTR) was defined as the total macroscopic removal of the tumor; and subtotal resection was defined as the subtotal macroscopic tumor removal. The estimation of the extent of tumor removal was validated by reviewing the postoperative MRI.

Pathological examination. All specimens underwent fixation in $4 \%$ neutral formalin $\left(24 \mathrm{~h}\right.$ at $\left.4^{\circ} \mathrm{C}\right)$, routine dehydration using a alcohol gradient series (ethanol $95 \%$ for $5 \mathrm{~min}$, ethanol $95 \%$ for $5 \mathrm{~min}$ and ethanol $80 \%$ for $5 \mathrm{~min}$ ), paraffin-embedding and slicing into $4-\mu \mathrm{m}$ sections, and then staining using hematoxylin-eosin (hematoxylin 1\%, eosin $0.5 \%$ ) at room temperature for $\sim 2 \mathrm{~h}$. Immunohistochemical staining was used for differential diagnoses. Immunohistochemistry was performed using the indirect immunoperoxidase technique. Bovine serum albumin (3\%) (Beijing Zhongshan Golden Bridge Biotechnology Co., Ltd.; OriGene Technologies, Beijing, China) was used for blocking at room temperature for $1 \mathrm{~h}$. Primary antibodies included pre-diluted monoclonal antibodies against glial fibrillary acidic protein (GFAP; cat. no. ZM-0118, Beijing Zhongshan Golden Bridge Biotechnology Co., Ltd.; OriGene Technologies; 1:200), nestin (cat. no. ZA-0628, 1:200) and epithelial membrane antigen (EMA; cat. no. ZM-0095, 1:200), Ki-67 antigen (cat. no. ZM-0165, 1:200), which were incubated for $12 \mathrm{~h}$ at $4^{\circ} \mathrm{C}$ and subsequently incubated with the HRP-conjugated mouse anti-human IgG (cat. no. SPN-9001; Beijing Zhongshan Golden Bridge Biotechnology Co., Ltd.; OriGene Technologies; $1: 100$ ) for $2 \mathrm{~h}$ at room temperature. The SuperPicture ${ }^{\mathrm{TM}}$ 3rd Gen IHC Detection kit (Invitrogen; Thermo Fisher Scientific, Inc., Waltham, MA, USA) was used to evaluate staining, according to the manufacturer's protocol. For antigen retrieval, slides were boiled in EDTA buffer $(\mathrm{pH}$ 8.0; cat. no. ZLI-9066; Beijing Zhongshan Golden Bridge Biotechnology Co., Ltd.; OriGene Technologies; Tris 30.27 g, EDTA $1.461 \mathrm{~g}$ and $\mathrm{H}_{2} \mathrm{O} 500 \mathrm{ml}$ ) under high pressure. Slides were counterstained with hematoxylin (1\%) at room temperature for $\sim 2 \mathrm{~h}$. An appropriate negative control (1 mmol/l PBS) was used. Quantitative evaluation of Ki-67 was obtained by calculating the percentage of Ki-67 positive nuclei in 100 tumor cells from the microscopic field (light microscope; 6 fields analyzed; magnification, x100; Beijing Konghai Science and Technology Development Co., Ltd., Beijing, China) with the highest density of labeled nuclei.

Follow-up data for all patients were obtained through individual office visits or telephone interviews performed every 6 months. Modified Japanese Orthopedic Association (JOA) scores (Table II) were used to assess neurological function. All patients were reassessed in June 2016 as a last clinical follow-up.

\section{Results}

Clinical presentation. The clinical features of the 8 patients are summarized in Table III. The mean age of patients at the

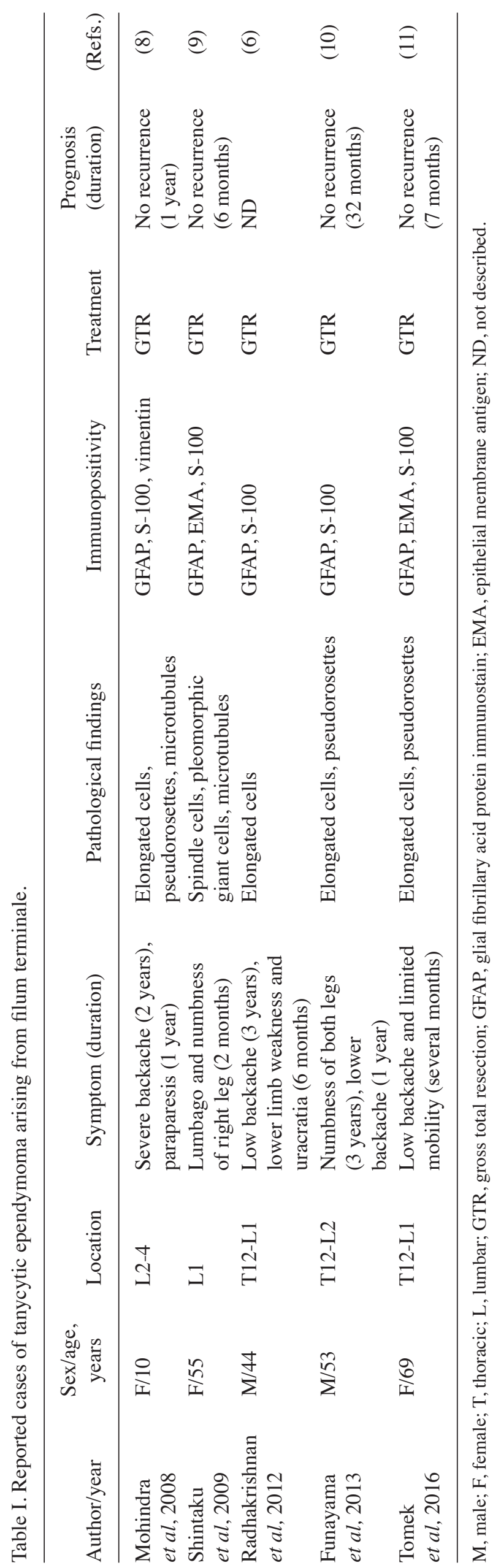


Table II. Modified Japanese Orthopedic Association scale (total score out of 17 points).

\begin{tabular}{ll}
\hline Section & $\begin{array}{l}\text { Score, } \\
\text { points }\end{array}$ \\
\hline
\end{tabular}

Motor function of upper extremity

Unable to feed oneself

Unable to use knife and fork; able to eat with a spoon

Able to use knife and fork with much difficulty

Able to use knife and fork with slight difficulty

Normal

Motor function of lower extremity

Unable to walk

Can walk on flat floor with walking aid

Can walk up and/or down stairs with handrail

Lack of stability and smooth gait

Normal

Sensory function of upper extremity

Severe sensory loss or pain

Mild sensory loss

Normal

Sensory function of lower extremity

Severe sensory loss or pain

Mild sensory loss

Normal

Sensory function of trunk extremity

Severe sensory loss or pain

Mild sensory loss

Normal

Bladder function

Unable to void

Marked difficulty in micturition (retention)

Difficulty in micturition (frequency, hesitation)

Normal

time of surgery was 40.5 years (range, 24-62 years). This series consisted of 1 male and 7 females. The duration of symptoms ranged from 2 weeks to 10 years. The most common clinical symptoms were pain and numbness of the waist and crura in 4 patients, and double crura in the other 4 patients. The mean preoperative JOA score was 12.6 (range, 10-14).

Radiological findings. Preoperative MRI results were available for all patients (Table III). On the MRI scans, lesions were oval or round in shape with well-defined boundaries and to the lesions were close to the spinal cord or cauda equina. On T1-weighted images (T1WI), lesions were isointense in 4 cases, iso- to hyperintense in 2 cases, and iso- to hypointense in 2 cases. On T2-weighted images (T2WI), the lesion was isointense in 5 cases, iso- to hypointense in 1 case, iso- to hyperintense in 1 cases, and hyperintense in 1 case. Contrast-enhanced T1WI revealed 3 cases with markedly homogeneous enhancement, 1 case with markedly heterogeneous enhancement, 3 cases with mild homogeneous enhancement and 1 case with mild heterogeneous enhancement. According to the preoperative MRI, no cases were diagnosed as ependymoma. The differential diagnosis included meningioma ( 3 cases, $37.5 \%$ ), schwannoma (4 cases, 50\%) and cholesteatoma (1 case, $12.5 \%$ ). Pre- and postoperative MRI for 2 patients (cases 3 and 4) are illustrated in Figs. 1 and 2.

Surgical and pathological findings. All patients underwent a posterior laminectomy approach, with GTR performed in 7 patients. Following opening the dura mater, the lesions were located in the cauda equina and appeared to arise from nerve roots or TE. Intraoperatively, the tumors typically appeared as moderate consistency, reddish-gray, solid masses. The tumors were closely adhered to the cauda equina, with moderate blood supply and a median intraoperative blood loss of $100 \mathrm{ml}$. In the fourth patient, total resection of the tumor failed due to its rich blood supply and close adhesion to the cauda equina. Histological examination of the tumor with hematoxylin and eosin staining revealed spindle cells with oval and elongated nuclei, termed tanycytes. Immunohistochemical staining demonstrated strong, positive staining of glial fibrillary acidic protein (GFAP), nestin and epithelial membrane antigen (EMA). The mindbomb homolog 1, also known as Ki-67 antigen, labeling index was $0.5-5 \%$, which indicated low proliferative activity. Ultrastructural examination demonstrated spindle cells arranged in bundles with scant extracellular matrix. Intercellular junctions, numerous slender surface microvilli and microvilli-lined lumina were observed (Fig. 3).

Postoperative evaluation. Following surgery, 5 patients experienced immediate improvement and 3 patients remained unchanged from their preoperative condition. All patients were postoperatively treated with neurotrophic drugs (Methylcobalamin, $1.5 \mathrm{mg}, 1$ tablet 3 times daily). In the 2-week and 3-month follow-up MRI, there was no evidence of tumor recurrence. During a mean follow-up period of 27 months, neurological status had markedly improved in 7 patients and was stable in 1 patient, compared with their postoperative neurological deficits, including pain or loss of sensation in the legs and uracratia (urinary incontinence, i.e., loss of urinary control ability). However, a number of patients complained of sensory deficit (2 cases, 25\%) and sphincter dysfunction (1 case, 12.5\%). During the final assessment, the mean JOA score was 16.6 (range, 16-17) for the 8 patients. The final mean JOA score was markedly improved compared with the preoperative mean JOA score. Surgical outcomes and assessment of neurological functions are summarized in Table III.

\section{Discussion}

Table I depicts a summary of the 5 reported cases of TE located in the filum terminale $(6,8-11)$. Of the 5 patients, 3 were female and 2 were male, 4 patients were adults ageing from 44-69 years old. The remaining patient was a 10 -year-old female child, which was reported by Mohindra et al in 2008 (8); the youngest patient with TE that has been reported, to the best of our knowledge. The predominant symptoms experiences by patients included backache, pain and numbness in the lower extremities. All 5 patients received complete 


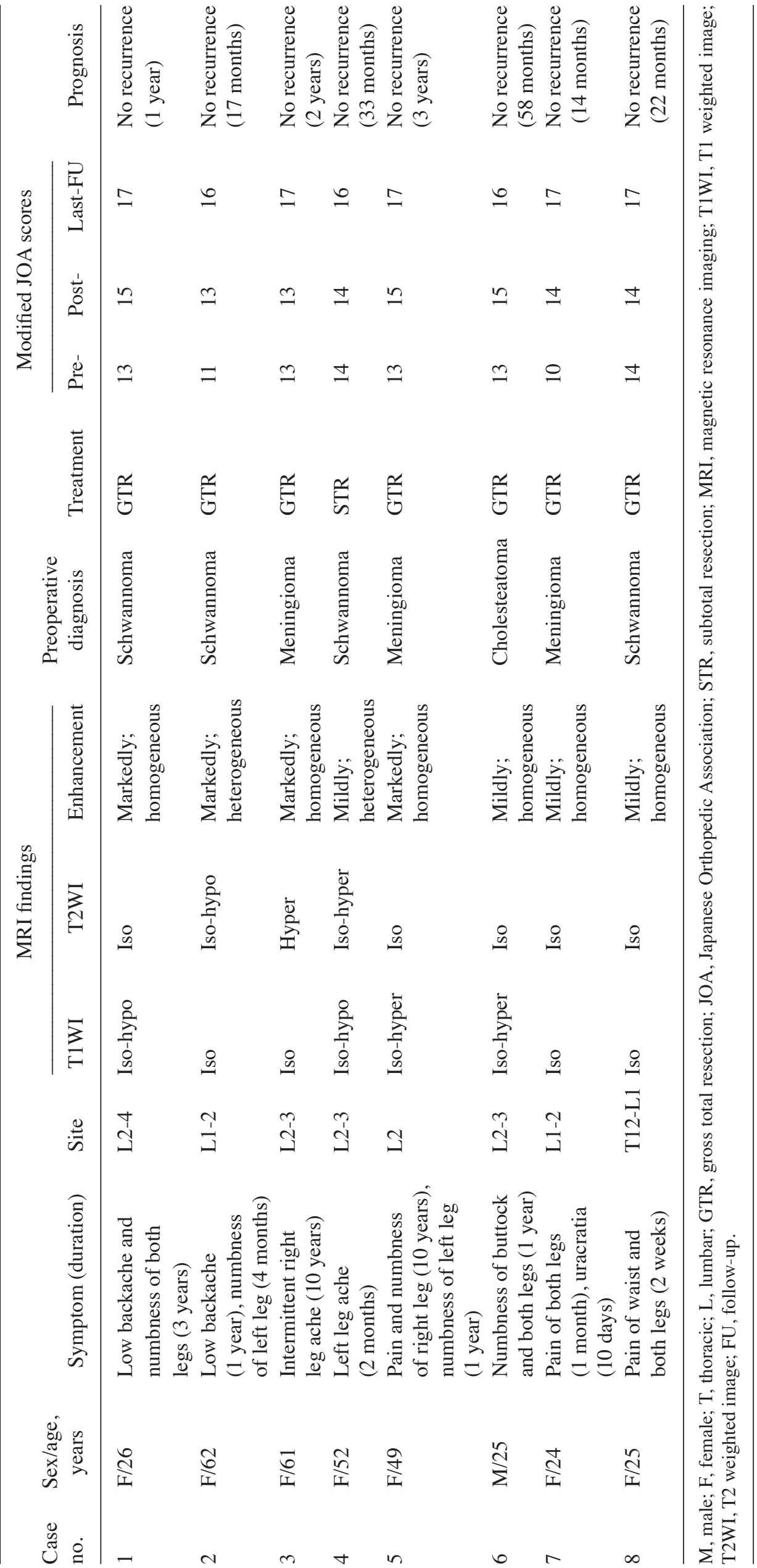



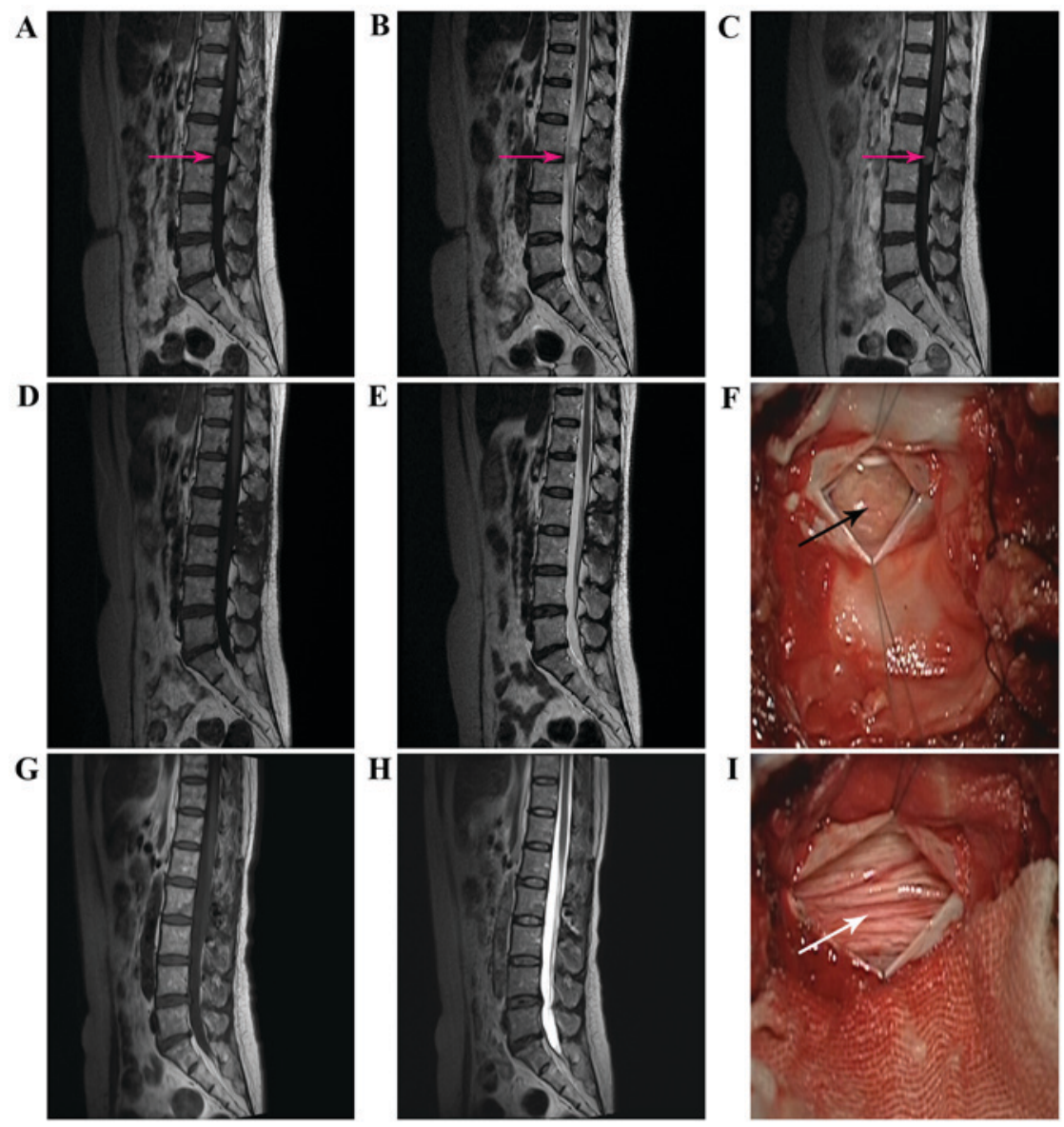

Figure 1. MRI of the spinal cord, for case 3, identified an isointense tumor on thoracic (A) T1- and (B) T2-weighted images. (C) Contrast-enhanced sagittal image identified a slightly hyperintense tumor with inhomogeneous enhancement. Sagittal T1- and T2-weighted images of the spine following laminectomies and tumor resection demonstrated gross total resection with no residual mass in the (D, T1; E, T2) 2-weekand (T1, G; and T2, H) 3-month follow-up MRI. The attached nerve rootlets were closely adhered to the (F) lesion (black arrow) and the (I) nerve rootlet (white arrow) of origin was transected to remove the lesion en bloc. Pink arrows indicate the tumor of the filum terminale. MRI, magnetic resonance imaging; T, thoracic; L, lumbar.
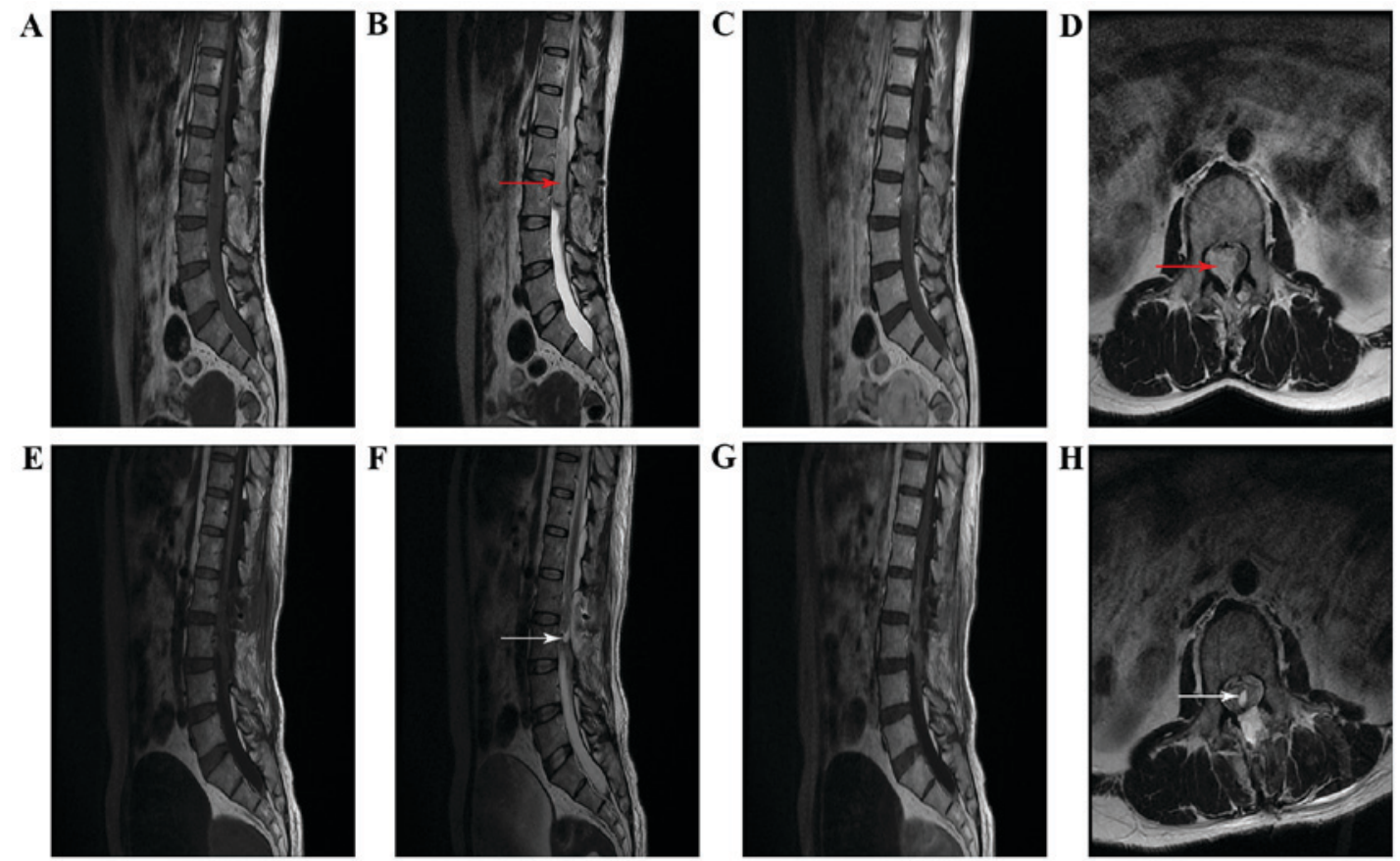

Figure 2. Spinal cord MRI scans of case 4. Sagittal (A) T1-weighted, (B) T2-weighted and (C, sagittal; D, coronal) contrast-enhanced T1-weighted MRI identified an isointense tumor with notable enhancement in T12-L2 (red arrow). Sagittal (E) T1- and (F) T2-weighted imaging, contrast-enhanced (G) T1-weighted image and $(\mathrm{H})$ coronal image revealed subtotal removal of the tumor, and an area of high signal identified the residual tumor (white arrow). MRI, magnetic resonance imaging; T, thoracic; L, lumbar. 

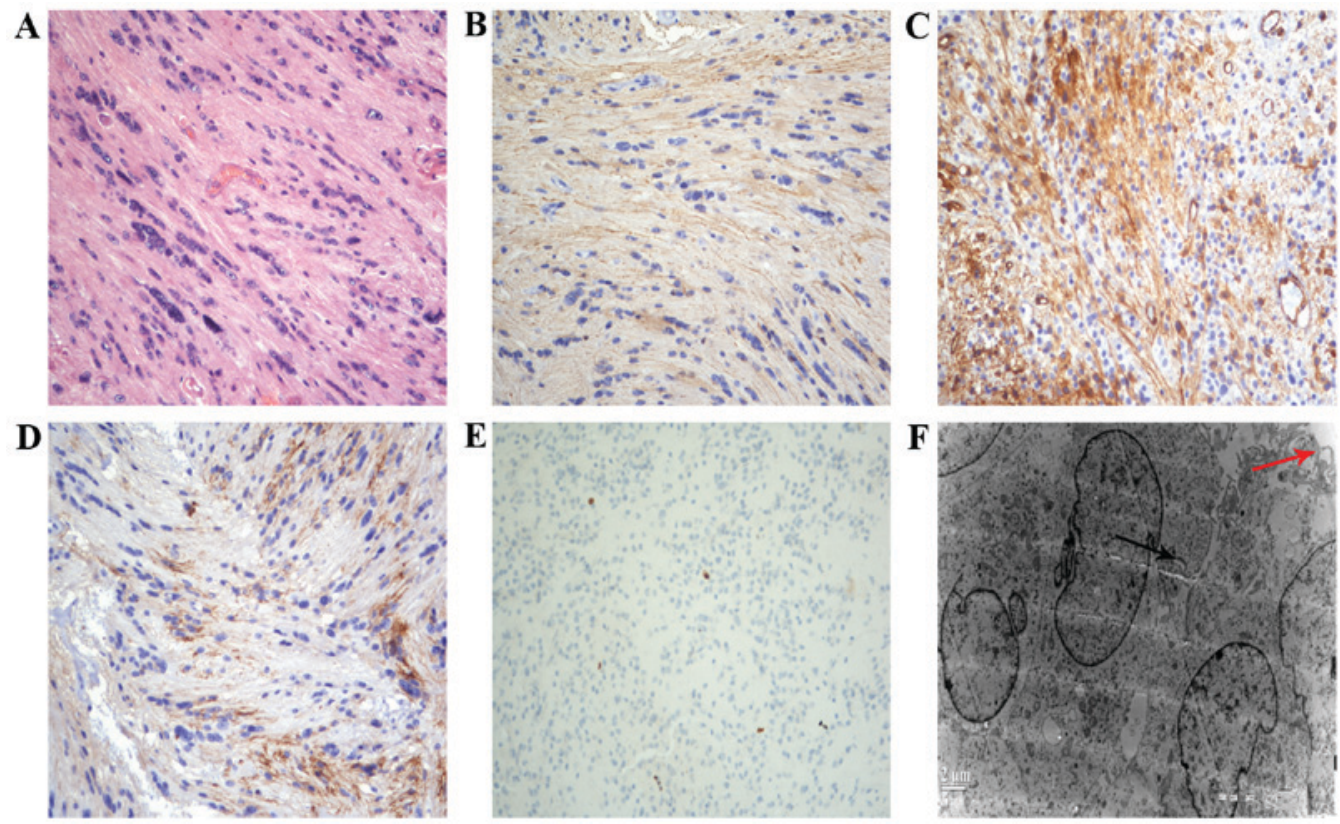

Figure 3. (A) Histological section exhibited spindle cell neoplasm of moderate cellularity arranged in interlacing fascicles. The tumor cells had discreetly elongated bipolar nuclei. The tumor cells were diffusely and strongly positive for (B) glial fibrillary acid protein immunostaining, (C) nest in and (D) epithelial membrane antigen. (E) The Ki-67 labeling index was low (2\%). (F) Electron micrograph of the tumor exhibited intercellular junctions (black arrow) and numerous slender surface microvilli (red arrow). Original magnification in (A-E) is x200, (F) is x6000.

surgical resection and postoperative pathology demonstrated that there were notable long spindle cells and pseudorosettes in the tumor, and immunohistochemical staining indicated that the tumor was positive for GFAP, EMA and S-100. With the exception of 1 case in which the prognosis of 1 patient (6) was not described, no recurrence was identified in the remaining 4 patients during the follow-up period.

TE may occur at any age, however the pathogenic sites vary for different ages. Pathogenic sites in children and adolescents are commonly in the cerebral cranium, whilst the majority of pathogenic sites in middle aged and elderly patients are in the spinal cord (11). With TE and spine as keywords, a search of the PUBMED database returned 27 articles (January 10th, 2017; https://www.ncbi.nlm.nih. gov/pmc/), in which 34 cases of spinal TE were reported in total $(2,3,6-30)$. By reviewing the material of these 34 cases of patients with spinal TE, it was revealed that the ages of the patients ranged from 10-76 years, with an average age of 40 years, and that the sex ratio of females to males was 1.25:1. The pathogenic sites were mainly in the cervical spinal cord (15 cases) and $\mathrm{T}$ cord (14 cases), whilst the remaining 5 cases occurred in the cauda equine and filum terminale. Combining the data from the present study and the 5 previous cases, it was identified that $10 / 13$ patients with TE of the filum terminale were female. The sex ratio of females to males was 10:3, which was greater than the sex ratio of all patients with spinal TE, indicating that TE of the filum terminale mostly affected females. The clinical manifestations of TE were associated with the pathogenic site of the tumor. For patients exhibiting TE in the filum terminale, the predominant clinical manifestations were backache, pain and numbness in the lower extremities, among others. In terms of the MRI scan, the tumor generally displayed isointense T1 signals and iso- or hyperintense T2 signals whose boundaries were relatively clear and would be strengthened significantly on contrast MRI scans.

The tissue origin of ependymoma has always been controversial (31), however the general opinion is that it originates in the radial glias, namely the tanycytes of ependyma and the precursor cells of neurogliocytes $(32,33)$. Ependymal cells, also termed periventricular cells, have long cell processes extending to the ependymal surface (28). In 1978, Friede and Pollak (3) first proposed TE, reporting6cases according to the characteristics of tumor cells similar to tanycytes. Previous studies on this particular tumor type suggested that it originated in the ependymal cells, and it has been classified as a subtype of ependymoma in the WHO classification of Tumors of the Central Nervous System in 2000 (7,26,34). The genetic characteristics of TE remain unclear; however, it has been reported in the literature that 2 cases were associated with neurofibromatosis type 2 and its tumor suppressor gene neurofibromin 2, which was inactivated; 1 case was potentially caused by family genetic ependymoma $(16,17,35)$.

The majority of TE diagnoses are based on its histological characteristics, notably the tanycyte and immunohistochemical characteristics (32). Electron microscopy is vital in diagnosing TE, due to the difficulty in differentiating it from similar tumors (8). The majority of the cases in this group were initially diagnosed as schwannoma or spinal meningioma by a preoperative imaging examination, and were postoperatively confirmed as misdiagnoses. In pathological diagnosis, improving the understanding of the histological morphology of this tumor is required. Schwannoma is a common spinal canal tumor, which is easy to confuse with TE due to its similar histological morphology of elongated cytoplasmic processes and absent atypia in nuclei. Schwannoma, in the spinal canal, always occurs extra-medullary spaces with well-defined boundaries. Histologically, its thick wall and hyalinized blood 
vessels are also important in differentiating these two tumors; in addition, schwannoma typically has an Antoni type A and B pattern. In routine pathological examination, a definite diagnosis can be achieved by immunohistochemically labeling for GFAP and S-100 proteins (36). Spinal meningioma is another example of a tumor that TE can be misidentified as. This tumor originates from arachnoid granulations, the majority of which are located subdurally-extra-medullarily, notably in the T vertebra; additionally, dural tail sign is occasionally present in spinal meningioma. This type of tumor is mainly composed of dural cells arranged in patches, or nest-like bulks, of different sizes, with a small amount of mesenchyme between the nest-like bulks. Dural cells are characterized by the following: A large volume; obscure boundaries presenting chimerism; abundant cytoplasm; large nuclei in round or oval shape; and clear nuclear membranes $(11,37)$. The final example of a tumor that TE can be misidentified as is subependymoma. This type of tumor, previously termed subependymal astrocytoma, frequently affects middle-aged and elderly people, with the majority of cases located in the fourth ventricle and lateral ventricles. Histologically, its clustered nuclei are embedded in a dense matrix of glial fibers and, along with the formation of numerous follicular cavities, these can be used as identifying factors in differentiating subependymoma from ependymoma $(6,7)$.

Currently, the primary treatment method for TE is complete surgical resection of the tumor $(2,38)$. For patients undergoing complete surgical resection, postoperative radiotherapy is not necessary $(11,26)$. For patients with postoperative recurrence, the treatments used are surgical resection with postoperative radiotherapy $(7,30)$. Of the patients investigated in the present study group, only 1 patient did not receive complete surgical resection of the tumor as the tumor had adhered firmly to the spinal cord and wrapped around the cauda equina. In order to decrease postoperative complications, a partial resection was opted for instead of a complete surgical resection. Therefore, the treatment principle for TE in the filum terminale is to carefully separate tumor boundaries intraoperatively, spare no effort to protect the cauda equina and strive to achieve complete surgical resection. The prognosis of TE is associated with the patient age, tumor site and the extent of surgical resection. It has been reported that the 5-year and 10-year survival rates for TE are improved compared with those with other subtypes of ependymoma $(33,39)$.

In conclusion, the current case series and literature review suggest that cauda equina TE should be taken into consideration when the tumors exhibit enhancement on MRI. TE is the rarest variant of ependymoma and its diagnosis largely depends on histopathological examination. Neurosurgeons should be aware of this lesion entity. Fortunately, cauda equina TE usually has a favorable prognosis, and GTR or STR are expected whenever possible.

\section{References}

1. Gilbert MR, Ruda R and Soffietti R: Ependymomas in adults Curr Neurol Neurosci Rep 10: 240-247, 2010.

2. Cepeda S, Hernández-Laín A, Munarriz PM, Martinez González MA and Lagares A: Spinal tanycytic ependymoma associated with neurofibromatosis type 2 . Clin. Neuropathol 33: 311-314, 2014.
3. Friede RL and Pollak A: The cytogenetic basis for classifying ependymomas. J Neuropathol Exp Neurol 37: 103-118, 1978.

4. Ragel BT, Townsend JJ, Arthur AS and Couldwell WT: Intraventricular tanycytic ependymoma: Case report and review of the literature. J Neurooncol 71: 189-193, 2005.

5. Liu Z, Li J, Liu Z, Wang Q, Famer P, Mehta A, Chalif D, Wang Y and Li JY: Supratentorial cortical ependymoma: Case series and review of the literature. Neuropathology 34: 243-252, 2014.

6. Radhakrishnan N, Nair NS, Hingwala DR, Kapilamoorthy TR and Radhakrishnan VV: Tanycytic ependymoma of filum terminale: A case report. Clin Neur Neurosurg 114: 169-171, 2012.

7. Ortiz Ydel M, Pérez Berenguer JL, Mercado Acosta J, Polo M, de Jesús-Garces $\mathrm{O}$ and Vega IE: Tanycytic ependymoma in a 76-year-old Puerto Rican male. Int J Clin Exp Pathol 7: 7789-7794, 2014.

8. Mohindra S, Bal A and Singla N: Pediatric tanycytic ependymoma of the cauda equina: Case report and review of the literature. J Child Neurol 23: 451-454, 2008.

9. Shintaku M and Sakamoto T: Tanycytic ependymoma of the filum terminale with pleomorphic giant cells. Brain Tumor Pathol 26: 79-82, 2009.

10. Funayama T, Sakane M, Yoshizawa T, Takeuchi Y and Ochiai N: Tanycytic ependymoma of the filum terminale associated with multiple endocrine neoplasia type 1: First reported case. Spine J 13: e49-e54, 2013.

11. Tomek M, Jayajothi A, Brandner S, Jaunmuktane Z, Lee $\mathrm{CH}$ and Davagnanam I: Imaging features of spinal tanycytic ependymoma. Neuroradiol J 29: 61-65, 2016.

12. Spaar FW, Blech M and Ahyai A: DNA-flow fluorescence-cytometry of ependymomas. Report on ten surgically removed tumours. Acta Neuropathol 69: 153-160, 1986.

13. Langford LA and Barré GM: Tanycytic ependymoma. Ultrastruct Pathol 21: 135-142, 1997.

14. Dvoracek MA and Kirby PA: Intraoperative diagnosis of tanycytic ependymoma: Pitfalls and differential diagnosis. Diagn Cytopathol 24: 289-292, 2001.

15. Kawano N, Yagishita S, Oka H, Utsuki S, Kobayashi I, Suzuki S, Tachibana S and Fujii K: Spinal tanycytic ependymomas. Acta Neuropathol 101: 43-48, 2001.

16. Kobata $\mathrm{H}$, Kuroiwa $\mathrm{T}$, Isono $\mathrm{N}$, Nagasawa $\mathrm{S}$, Ohta $\mathrm{T}$ and Tsutsumi A: Tanycytic ependymoma in association with neurofibromatosis type 2. Clin Neuropathol 20: 93-100, 2001.

17. Ueki K, Sasaki T, Ishida T and Kirino T: Spinal tanycytic ependymoma associated with neurofibromatosis type 2-case report. Neurol Med Chir (Tokyo) 41: 513-516, 2001.

18. Boccardo M, Telera S and Vitali A: Tanycytic ependymoma of the spinal cord. Case report and review of the literature. Neurochirurgie 49: 605-610, 2003.

19. Ito T, Ozaki Y, Nakagawara J, Nakamura $H$, Tanaka $S$ and Nagashima K: A case of cervicomedullary junction tanycytic ependymoma associated with marked cyst formation. Brain Tumor Pathol 22: 29-33, 2005.

20. Sato K, Kubota T, Ishida M and Handa Y: Spinal tanycytic ependymoma with hematomyelia-case report. Neurol Med Chir (Tokyo) 45: 168-171, 2005.

21. Shintaku M, Nagata N and Itoh H: Tanycytic ependymoma of the spinal cord with anaplastic cytological features. Brain Tumor Pathol 26: 7-10, 2009.

22. Du J, Zhou X, Tang Q, Ma H, Zhou H, Wang J, Lu Z and Yin H: Tanycytic ependymoma: Two case reports and review of the literature. Comp Clin Pathol 18: 449-453, 2009.

23. Lim BS, Park SQ, Chang UK and Kim MS: Spinal cord tanycytic ependymoma associated with neurofibromatosis type 2 . J Clin Neurosci 17: 922-924, 2010.

24. Ishihama $H$, Nakamura $M$, Funao $H$, Ishii $K$, Matsumoto $M$, Toyama Y and Chiba K: A rare case of spinal dumbbell tanycytic ependymoma. Spine (Phila Pa 1976) 36: E612-E614, 2011.

25. Tosun O, Turkoglu OF, Ozmen EK, Onursever A and Arslan H: Spinal tanycytic ependymoma with diffusion restriction on MRI. Acta Neurol Belg 112: 77-80, 2012.

26. Krisht KM and Schmidt MH: Tanycytic ependymoma: A challenging histological diagnosis. Case Rep Neurol Med 2013: 170791, 2013

27. Kuga Y, Ohnishi H, Kodama Y, Takakura S, Hayashi M, Yagi R, Matsushima K, Okamoto K, Taomoto K and Takahashi H: Cerebral and spinal cord tanycytic ependymomas in a young adult with a mutation in the NF2 gene. Neuropathology 34: 406-413, 2014.

28. Furlan JC, Chui MH, Croul SE and Kongkham P: Mystery case: Tanycytic ependymoma of the conus medullaris: A rare cause of low back pain. Neurology 82: e212-e213, 2014. 
29. Maugeri R, Giugno A, Graziano F, Visocchi M, Giller C and Iacopino DG: Delayed chronic intracranial subdural hematoma complicating resection of a tanycytic thoracic ependymoma. Surgical Neurol Int 7 (Suppl 1): S20-S22, 2016.

30. Singla N, Kapoor A, Radotra BD and Chatterjee D: Tanycytic ependymoma of cervical cord presenting with spontaneous intratumoral hemorrhage. Spine J 16: e733-e734, 2016.

31. Rigante L, Novello M, Massimi L and Caldarelli M: A cortical cystic epileptogenic lesion: Tanycytic ependymoma. Acta Neurol Belg 113: 523-525, 2013.

32. Divito A, Keller JT, Hagen M and Zuccarello M: Vestibular schwannoma or tanycytic ependymoma: Immunohistologic staining reveals. Surg Neurol Int 5: 158, 2014.

33. Agarwal S, Stevenson ME, Sughrue ME, Wartchow EP Mierau GW and Fung KM: Features of intraventricular tanycytic ependymoma: Report of a case and review of literature. Int J Clin Exp Pathol 7: 3399-3407, 2014.

34. Radner H, Blümcke I, Reifenberger G and Wiestler OD: The new WHO classification of tumors of the nervous system 2000. Pathologe 23: 260-283, 2002 (In German).
35. Dimopoulos VG, Fountas KN and Robinson JS: Familial intracranial ependymomas. Report of three cases in a family and review of the literature. Neurosurg Focus 20: E8, 2006.

36. Erdogan E, Ongürü O, Bulakbasi N, Baysefer A, Gezen F and Timurkaynak E: Schwannoma of the lateral ventricle: Eight-year follow-up and literature review. Minim Invasive Neurosurg 46: 50-53, 2003.

37. Shukla S, Malhotra KP, Awasthi NP, Husain N and Singh SK Intraventricular tanycytic ependymoma: An uncommon fibrillary variant. Neurol India 62: 200-201, 2014.

38. Kambe A, Kurosaki M, Watanabe T and Nakazato Y: Pediatric supratentorial cortical tanycytic ependymoma associated with absence seizures. Clin Neuropathol 33: 308-310, 2014.

39. Zhang S, Wang X, Zhang Z and Chen Y: Tanycytic ependymoma arising from the right lateral ventricle: A case report and review of the literature. Neuropathology 28: 427-432, 2008.

This work is licensed under a Creative Commons Attribution-NonCommercial-NoDerivatives 4.0 International (CC BY-NC-ND 4.0) License. 